\title{
A computationally efficient method for hand-eye calibration
}

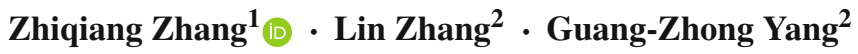

Received: 13 April 2017 / Accepted: 10 July 2017 / Published online: 19 July 2017

(C) The Author(s) 2017. This article is an open access publication

\begin{abstract}
Purpose Surgical robots with cooperative control and semiautonomous features have shown increasing clinical potential, particularly for repetitive tasks under imaging and vision guidance. Effective performance of an autonomous task requires accurate hand-eye calibration so that the transformation between the robot coordinate frame and the camera coordinates is well defined. In practice, due to changes in surgical instruments, online hand-eye calibration must be performed regularly. In order to ensure seamless execution of the surgical procedure without affecting the normal surgical workflow, it is important to derive fast and efficient hand-eye calibration methods.

Methods We present a computationally efficient iterative method for hand-eye calibration. In this method, dual quaternion is introduced to represent the rigid transformation, and a two-step iterative method is proposed to recover the real and dual parts of the dual quaternion simultaneously, and thus the estimation of rotation and translation of the transformation.

Results The proposed method was applied to determine the rigid transformation between the stereo laparoscope and the robot manipulator. Promising experimental and simulation results have shown significant convergence speed improvement to 3 iterations from larger than 30 with regard to standard optimization method, which illustrates the effectiveness and efficiency of the proposed method.
\end{abstract}

Keywords Minimally invasive surgery $\cdot$ Robot-camera calibration · Hand-eye calibration · Optimization

Zhiqiang Zhang

z.zhang3@leeds.ac.uk

1 School of Electronic and Electrical Engineering, University of Leeds, Leeds, UK

2 Hamylyn Centre, Imperial College London, London, UK

\section{Introduction}

With increasing maturity of master-slave surgical robots, research in robotically assisted minimally invasive surgery has now focussed on the development of cooperative control and automation of certain repetitive surgical steps such as suturing, ablation, and microscopic image scanning [13]. This is beneficial for the operating surgeon who can share control with the robot on low-level surgical maneuvers, freeing both perceptual and cognitive capacity on more demanding tasks that require direct human interaction [4]. Thus far, the use of vision guidance augmented with pre- and intra-operative imaging such as CT, MR and ultrasound has been applied to a range of surgical tasks including neurosurgery, orthopaedics, and cardiothoracic interventions [5-9]. However, in order to perform effective image-guided interventions, it is essential to recover the transformation between the robot coordinate frame and the endoscopic camera coordinates, which is a well-known hand-eye calibration problem in robotics.

In general, any rigid transformation can be described by two parameters: a translation vector and a rotation matrix. Thus far, a number of closed-form solutions have been proposed for hand-eye calibration. For example, Shiu and Ahmad [10] proposed to solve the robot-sensor calibration problem by estimating the orientational component and translational component separately. The orientation component was derived by utilizing the angle-axis formulation of rotation, while the translational component could be solved using standard linear system techniques once the rotational part is estimated. To simplify the process, Park and Martin [11] proposed a solution for the orientation component by taking advantage of Lie group theory to transform the orientation component into a linear system. Chou and Kamel [12] introduced quaternion to represent orientation 
and re-formulated the determination of the rotation matrix as a homogeneous linear least squares problem. Closedform solution was then derived using the generalized inverse method with singular value decomposition analysis. Alternatively, Liang and Mao [13] applied the Kronecker product to get the homogeneous linear equation for the rotation matrix. Pan et al. [14] and Najifi et al. [15] also presented similar work. However, all the aforementioned methods separated the orientation component from the positional component, and errors in the rotation estimation could be propagated into the translational estimations.

Simultaneous solutions for rotational and translational components were also presented in the past decades. For instance, $\mathrm{Lu}$ and Chou [16] derived an eight-space formulation based on quaternion to obtain the least squares solution for the hand-eye calibration problem using the Gaussian elimination and Schur decomposition analysis. Daniilidis [17] introduced the idea of dual quaternion parameterization, which facilitated a new simultaneous solution for the hand-eye rotation and translation using singular value decomposition. Zhao and Liu [18] employed the screw motion theory to establish a hand-eye matrix equation by using quaternion, resulting in a simultaneous result for rotation and translation by solving linear equations. Andreff et al. [19] applied the Kronecker product to re-formulate the robot-sensor problem into a linear system. Least square solutions were derived to simultaneously solve the robot-sensor problem. Although all these methods can estimate rotational and translational components for the hand-eye calibration problems simultaneously, many of them involve complicated derivations.

To circumvent this problem, researchers tend to move from closed-form solutions to iterative methods due to its high efficiency and simplicity. The basic idea of iterative method is to minimize the difference between the left and right parts of the hand-eye equation or its variations. Thus far, a number of solutions have been proposed. For instance, Schmidt et al. [20] constructed a cost function using dual quaternion and quaternion multiplication properties, and classic optimization method was then applied to minimize the cost function. Strobl and Hirzinger [21] proposed a weighting schemes to construct a cost function. Similarly, they also applied classic nonlinear optimization method to solve the cost function minimization problem. Mao et al. [22] applied Kronecker product to establish a nonlinear objective function and then derived the iterative Jacobian formula to find the optimized solution using Gauss-Newton method or Levenberg-Marquet method. Zhao [23] proposed to use convex optimization, which can solve the hand-eye calibration problem in the form of a global linear optimization without starting values. Ruland et al. [24] proposed to integrate the hand-eye calibration problem into a branch-and-bound parameter space search. The presented method constituted the first guaranteed globally optimal estimator for simultaneous optimization of both components with respect to a cost function based on re-projection errors. Ackerman et al. [25] presented a unified algorithm which used gradient descent optimization on the Euclidean group. They also applied filtering to update the calibration parameters online based on new incoming data. Heller et al. [26,27] presented several formulations of hand-eye calibration that led to multivariate polynomial optimization problems. Convex linear matrix inequality (LMI) relaxation was used to effectively solve these problems and to obtain globally optimal solutions. Wu et al. [28] and Sarrazin et al. [29] also presented similar ideas for hand-eye calibration between robotic system and vision/ultrasound systems. All these methods can solve the hand-eye calibration problem, albeit being complex to implement in practice and involving complicated calculations. It also results in low efficiency, which may be not applicable to scenarios where online hand-eye calibration must be performed regularly.

In practice, the hand-eye calibration may need to be done frequently due to changes in surgical instruments and in the case of using pickup probes, their position related to the end effector is not always the same. Figure 1 shows an example of re-projection of da Vinci surgical instruments on laparoscopic images. The top left frame illustrates the re-projection right after the calibration where the error is minimal. As the instruments are moved away, the re-projection (shown in red) errors start to build up; therefore, online hand-eye calibration must be performed regularly. In order to ensure seamless execution of the surgical procedure without affecting the normal surgical workflow, it is important to derive a computationally efficient iterative method for solving hand-eye calibration. The contributions of the paper include:

- We proposed a two-step iterative method that can recover the real part and dual part of the dual quaternion efficiently and fast;

- We theoretically proved the convergence of the proposed two-step iteration method.

The proposed method was applied to determine the rigid transformation between the stereo laparoscope and the robot manipulator. Promising experimental and simulation results have shown significant convergence speeds improvement to 3 iterations from larger than 30 with regard to standard optimization method, which illustrates the effectiveness and efficiency of the proposed method.

The rest of the paper is organized as follows. The mathematical details of hand-eye calibration are given in "Problem statement" section. The proposed two-step iterative method is then introduced in "Two-step iterative method" section. Experimental results and conclusions are provided in "Exper- 

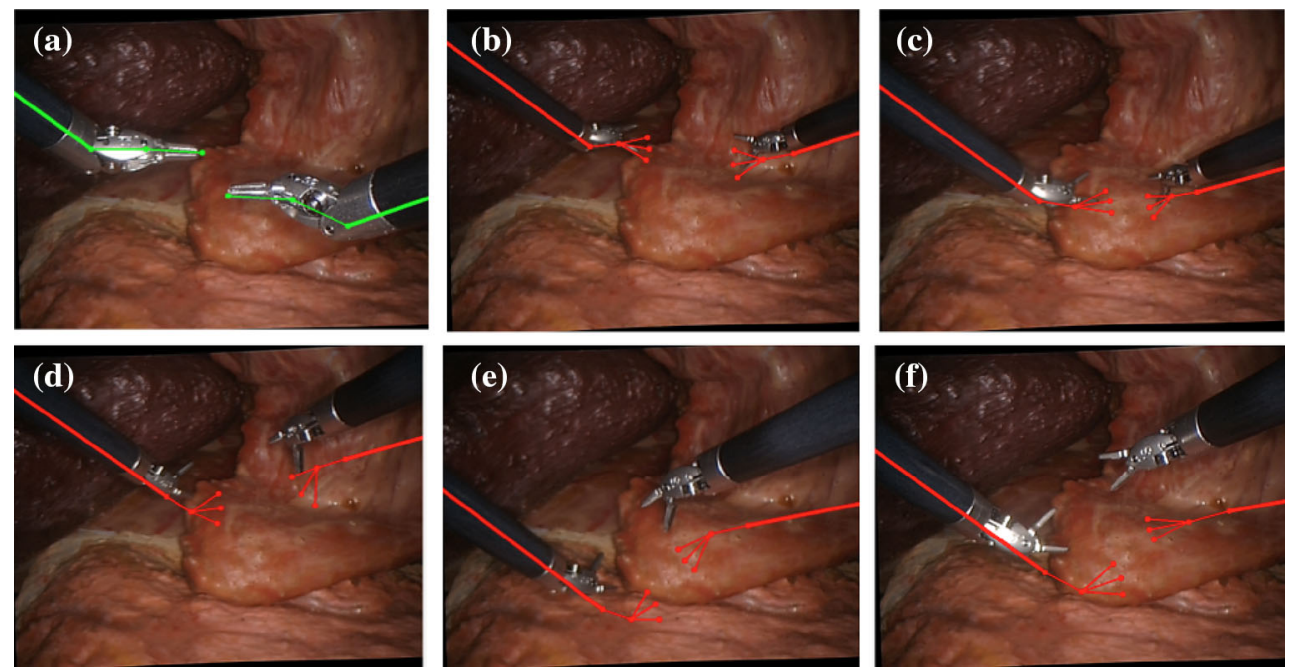

Fig. 1 An example of re-projection of da Vinci surgical instruments by using kinematic re-projection of the model directly onto laparoscopic images. a The re-projection right after the calibration; $\mathbf{b}-\mathbf{f}$ as time progresses and the instruments are moved away, the re-projection (shown in red) errors start to build up. Real-time hand-eye calibration is therefore required to re-estimate the transformation between the robot coordinate frame and the camera coordinates imental and simulation results and Conclusion" sections, respectively.

\section{Problem statement}

The most common way to describe the hand-eye calibration problem is using the homogeneous transformation matrix as:

$$
A X=X B
$$

where $A$ and $B$ are known homogeneous matrices, and $X$ is the unknown transformation between the robot coordinate frame and camera coordinate frame. For each homogeneous matrix, it is in the form of

$$
\left[\begin{array}{ll}
R & t \\
0 & 1
\end{array}\right]
$$

where $R$ is a $3 \times 3$ rotational matrix, and $t$ is a $3 \times 3$ translational vector. Thus, we can expand Eq. (1) as

$\left[\begin{array}{cc}R_{A} & t_{A} \\ 0 & 1\end{array}\right] \cdot\left[\begin{array}{cc}R_{X} & t_{X} \\ 0 & 1\end{array}\right]=\left[\begin{array}{cc}R_{X} & t_{X} \\ 0 & 1\end{array}\right] \cdot\left[\begin{array}{cc}R_{B} & t_{B} \\ 0 & 1\end{array}\right]$

where $R_{A}, R_{X}$ and $R_{B}$ are the rotational matrix parts of $A, X$ and $B$, and $t_{A}, t_{X}$ and $t_{B}$ are the translational parts, respectively. Equation (3) can be further simplified as:

$$
\left[\begin{array}{cc}
R_{A} R_{X} & R_{A} t_{X}+t_{A} \\
0 & 1
\end{array}\right]=\left[\begin{array}{cc}
R_{X} R_{B} & R_{X} t_{B}+t_{X} \\
0 & 1
\end{array}\right] .
$$

The purpose of hand-eye calibration is to find $R_{X}$ and $t_{X}$ given $J$ pair of $A_{i}$ and the corresponding $B_{i}$, where $i=$ $1,2 \ldots J$. We can have the estimation as:

$$
\left\{\hat{R}_{X}, \hat{t}_{X}\right\}=\underset{R_{X}, t_{X}}{\operatorname{argmin}}\left\{f\left(R_{X}, t_{X}\right)\right\}
$$

subject to

$$
R_{X} \cdot R_{X}^{\mathrm{T}}=I
$$

and

$\operatorname{det}\left(R_{X}\right)=1$

where $I$ is the identify matrix of order 3 , and $\operatorname{det}(\cdot)$ is the determinant of a $3 \times 3$ matrix. The cost function $f\left(R_{X}, t_{X}\right)$ can be defined as:

$f\left(R_{X}, t_{X}\right)=\sum_{i=1}^{J}\left\|A_{i} X-X B_{i}\right\|$

or

$$
\begin{aligned}
& f\left(R_{X}, t_{X}\right) \\
& \quad=\sum_{i=1}^{J}\left\{\left\|R_{A_{i}} R_{X}-R_{X} R_{B_{i}}\right\|+\left\|R_{A_{i}} t_{X}+t_{A_{i}}-R_{X} t_{B_{i}}-t_{X}\right\|\right\}
\end{aligned}
$$

where $\|\cdot\|$ is the Frobenius norm. Many algorithms, such as active set algorithm [30], interior point algorithm [31], sequential quadratic programming (SQP) algorithm [32] and 
so on, have been proposed so far to solve the above constrained minimization problem, but these methods tend to calculate the Jacobian matrix and Hessian matrix, which are computationally expensive. In the next section, we will present a simple two-step iterative method to solve the above constrained optimization problem.

\section{Two-step iterative method}

In order to convert the hand-eye calibration from the homogeneous transformation matrix format into dual quaternion representation, a short introduction of quaternion is therefore given at the beginning of this section.

\section{Quaternion and dual quaternion}

According to the Euler's rotation theorem, any rotation can be expressed as a unit quaternion $q$. Given any two unit quaternions $p=\left(p_{0}, v_{p}\right)$ and $q=\left(q_{0}, v_{q}\right)$, the multiplication of $p$ and $q$ can be represented as $[33,34]$ :

$p \otimes q=\mathcal{R}(q) p$

or

$p \otimes q=\mathcal{L}(p) q$

where $\otimes$ represents quaternion multiplication,

$\mathcal{R}(q)=\left[\begin{array}{cc}q_{0} & -v_{q}^{\mathrm{T}} \\ v_{q} & q_{0} \cdot I+\left\lfloor v_{q} \times\right\rfloor\end{array}\right]$

and

$\mathcal{L}(p)=\left[\begin{array}{cc}p_{0} & -v_{p}^{\mathrm{T}} \\ v_{p} & p_{0} \cdot I-\left\lfloor v_{p} \times\right\rfloor\end{array}\right]$.

Here, $\lfloor x\rfloor$ is the skew-symmetric matrix/cross-product operator.

In practice, quaternion can only be used to represent orientation. In order to represent orientation and translation together, quaternion is combined with dual number theory to form dual quaternion [35]. Each dual quaternion consists of two quaternions as:

$\breve{q}=q_{r}+q_{d} \varepsilon$

where $\varepsilon$ is the dual number, $q_{r}$ and $q_{d}$ are quaternion. Given any two dual quaternion $\breve{p}=p_{r}+p_{d} \varepsilon$ and $\breve{q}$, the multiplication can be defined as:

$\breve{q} \otimes \breve{p}=q_{r} \otimes p_{r}+\left(q_{r} \otimes p_{d}+q_{d} \otimes p_{r}\right) \varepsilon$.
For any homogeneous transformation in the form of

$\left[\begin{array}{ll}R & t \\ 0 & 1\end{array}\right]$

the corresponding dual quaternion representation $\breve{q}$ can be defined as:

$\left\{\begin{array}{l}q_{r}=D 2 q(R) \\ q_{d}=\frac{1}{2} q_{r} \otimes t\end{array}\right.$

where $D 2 q(\cdot)$ is the operator to convert a rotation matrix to the corresponding unit quaternion, and $t$ is taken as a pure quaternion with 0 scale part $[34,35]$.

\section{Two-step iteration}

For any $\mathrm{i}(i=1,2 \ldots J)$, we have

$A_{i} X=X B_{i}$.

Denote

$$
\begin{aligned}
\breve{q}_{A_{i}} & =q_{r, A_{i}}+q_{d, A_{i}} \varepsilon \\
\breve{q}_{B_{i}} & =q_{r, B_{i}}+q_{d, B_{i}} \varepsilon \\
\breve{q}_{X} & =q_{r, X}+q_{d, X} \varepsilon
\end{aligned}
$$

as the qual-quaternion representation for $A_{i}, B_{i}$ and $X$, respectively, Eq. (18) can thus be written as:

$\breve{q}_{X} \otimes \breve{q}_{A_{i}}=\breve{q}_{B_{i}} \otimes \breve{q}_{X}$.

According to Eq. (15), the above equation can be expanded as:

$q_{r, X} \otimes q_{r, A_{i}}+\left(q_{r, X} \otimes q_{d, A_{i}}+q_{d, X} \otimes q_{r, A_{i}}\right) \varepsilon$

$=q_{r, B_{i}} \otimes q_{r, X}+\left(q_{r, B_{i}} \otimes q_{d, X}+q_{d, B_{i}} \otimes q_{r, X}\right) \varepsilon$.

Therefore, we can have

$q_{r, X} \otimes q_{r, A_{i}}=q_{r, B_{i}} \otimes q_{r, X}$

and

$q_{r, X} \otimes q_{d, A_{i}}+q_{d, X} \otimes q_{r, A_{i}}=q_{r, B_{i}} \otimes q_{d, X}+q_{d, B_{i}} \otimes q_{r, X}$

According to Eqs. (10) and (11), Eq. (22) can then be written as:

$\left(\mathcal{L}\left(q_{r, B_{i}}\right)-\mathcal{R}\left(q_{r, A_{i}}\right)\right) q_{r, X}=0$

and Eq. (23) can be written as:

$\left(\mathcal{R}\left(q_{d, A_{i}}\right)-\mathcal{L}\left(q_{d, B_{i}}\right)\right) q_{r, X}=\left(\mathcal{L}\left(q_{r, B_{i}}\right)-\mathcal{R}\left(q_{r, A_{i}}\right)\right) q_{d, X}$. 
Therefore, we can have:

$H_{l, i} q_{r, X}=H_{r, i} q_{d, X}$

where

$H_{l, i}=\left[\begin{array}{l}\mathcal{L}\left(q_{r, B_{i}}\right)-\mathcal{R}\left(q_{r, A_{i}}\right) \\ \mathcal{R}\left(q_{d, A_{i}}\right)-\mathcal{L}\left(q_{d, B_{i}}\right)\end{array}\right]$

and

$H_{r, i}=\left[\begin{array}{c}0_{4 \times 4} \\ \mathcal{L}\left(q_{r, B_{i}}\right)-\mathcal{R}\left(q_{r, A_{i}}\right)\end{array}\right]$.

Define

$H_{l}=\left[\begin{array}{c}H_{l, 1} \\ H_{l, 2} \\ \vdots \\ H_{l, J}\end{array}\right]$

and

$H_{r}=\left[\begin{array}{c}H_{r, 1} \\ H_{r, 2} \\ \vdots \\ H_{r, J}\end{array}\right]$

we can have

$H_{l} \cdot q_{r, X}=H_{r} \cdot q_{d, X}$

and given any initial value for $q_{r, X}$ as $q_{r, X}^{0}, q_{r, X}$ and $q_{d, X}$ can be estimated as:

1. Set index $n=1$;

2. Calculate $q_{d, X}^{n}$ as:

$$
q_{d, X}^{n}=H_{r}^{+} \cdot H_{l} \cdot q_{r, X}^{n-1}
$$

where $(\cdot)^{+}$is the pseudo-inverse operator.

3. Calculate $q_{r, X}^{n}$ as

$$
q_{r, X}^{n}=H_{l}^{+} \cdot H_{r} \cdot q_{d, X}^{n} \cdot
$$

4. Set $n=n+1$ and repeat steps $2-4$ until $q_{r, X}^{n}$ and $q_{d, X}^{n}$ converge.

5. Re-scale the magnitudes and set the $q_{r, X}$ and $q_{d, X}$ estimates as

$$
\begin{aligned}
\hat{q}_{r, X} & =\frac{q_{r, X}^{n}}{\left\|q_{r, X}^{n}\right\|} \\
\hat{q}_{d, X} & =\frac{q_{r, X}^{n}}{\left\|q_{r, X}^{n}\right\|} q_{d, X}^{n} .
\end{aligned}
$$

6. The estimation of $X$ can thus be calculated as:

$$
\hat{X}=\left[\begin{array}{cc}
q 2 D\left(\hat{q}_{r, X}\right) & 2 \cdot\left(\hat{q}_{r, X}\right)^{*} \otimes \hat{q}_{d, X} \\
0 & 1
\end{array}\right]
$$

where $q 2 D(\cdot)$ is the operator to convert a unit quaternion to the corresponding rotation matrix and * represents the quaternion conjugate. To make the dimension of $X$ correct, only the vector part of quaternion $2 \cdot\left(\hat{q}_{r, X}\right)^{*} \otimes \hat{q}_{d, X}$ is selected in the above equation [34].

Theorem 1 The $q_{r, X}^{n}$ and $q_{d, X}^{n}$ can always converge to obtain the ground truth for $q_{r, X}$ and $q_{d, X}$ via the two-step iteration methods.

Proof Similar to Eq. (5), the purpose of the two-step iteration is to minimize

$$
\left\|H_{l} \cdot q_{r, X}-H_{r} \cdot q_{d, X}\right\|
$$

which means that $q_{r, X}^{n}$ and $q_{d, X}^{n}$ can converge to obtain the ground truth for $q_{r, X}$ and $q_{d, X}$ only if:

$$
\left\|H_{l} \cdot q_{r, X}^{n}-H_{r} \cdot q_{d, X}^{n+1}\right\| \leqslant\left\|H_{l} \cdot q_{r, X}^{n}-H_{r} \cdot q_{d, X}^{n}\right\|
$$

and

$$
\left\|H_{l} \cdot q_{r, X}^{n}-H_{r} \cdot q_{d, X}^{n}\right\| \leqslant\left\|H_{l} \cdot q_{r, X}^{n-1}-H_{r} \cdot q_{d, X}^{n}\right\| .
$$

For Eq. (37), we can have

$$
\begin{aligned}
& \left\|H_{l} \cdot q_{r, X}^{n}-H_{r} \cdot q_{d, X}^{n+1}\right\| \\
& =\left\|H_{l} \cdot q_{r, X}^{n}-H_{r} \cdot H_{r}^{+} \cdot H_{l} \cdot q_{r, X}^{n}\right\| \\
& =\left\|\left(I-H_{r} \cdot H_{r}^{+}\right) \cdot H_{l} \cdot q_{r, X}^{n}\right\|
\end{aligned}
$$

For any matrices $\Upsilon$ and $\Psi,\left\|I-\Upsilon^{+} \Upsilon\right\|<\left\|I-\Psi^{+} \Upsilon\right\|$ is always satisfied unless $\Upsilon=\Psi$ [36], so

$$
\begin{aligned}
& \left\|H_{l} \cdot q_{r, X}^{n}-H_{r} \cdot q_{d, X}^{n+1}\right\| \\
& \leqslant\left\|\left(I-H_{r} \cdot H_{r}^{+} \cdot H_{l} \cdot q_{r, X}^{n-1}\left(H_{l} \cdot q_{r, X}^{n}\right)^{+}\right) H_{l} \cdot q_{r, X}^{n}\right\| \\
& =\left\|H_{l} \cdot q_{r, X}^{n}-H_{r} \cdot H_{r}^{+} \cdot H_{l} \cdot q_{r, X}^{n-1}\right\| \\
& =\left\|H_{l} \cdot q_{r, X}^{n}-H_{r} \cdot q_{d, X}^{n}\right\| .
\end{aligned}
$$

For Eq. (38), we can also have

$$
\begin{aligned}
& \left\|H_{l} \cdot q_{r, X}^{n}-H_{r} \cdot q_{d, X}^{n}\right\| \\
& =\left\|H_{l} \cdot H_{l}^{+} \cdot H_{r} \cdot q_{d, X}^{n}-H_{r} \cdot q_{d, X}^{n}\right\| \\
& =\left\|\left(H_{l} \cdot H_{l}^{+}-I\right) H_{r} \cdot q_{d, X}^{n}\right\|
\end{aligned}
$$


Fig. 2 The hand-eye data were collected using the da Vinci Research Kit (DVRK): the pose of the tool in the robot base coordinate was obtained via the robot forward kinematics, while the corresponding pattern pose in the camera coordinate was derived using a pair of stereo images containing the pattern
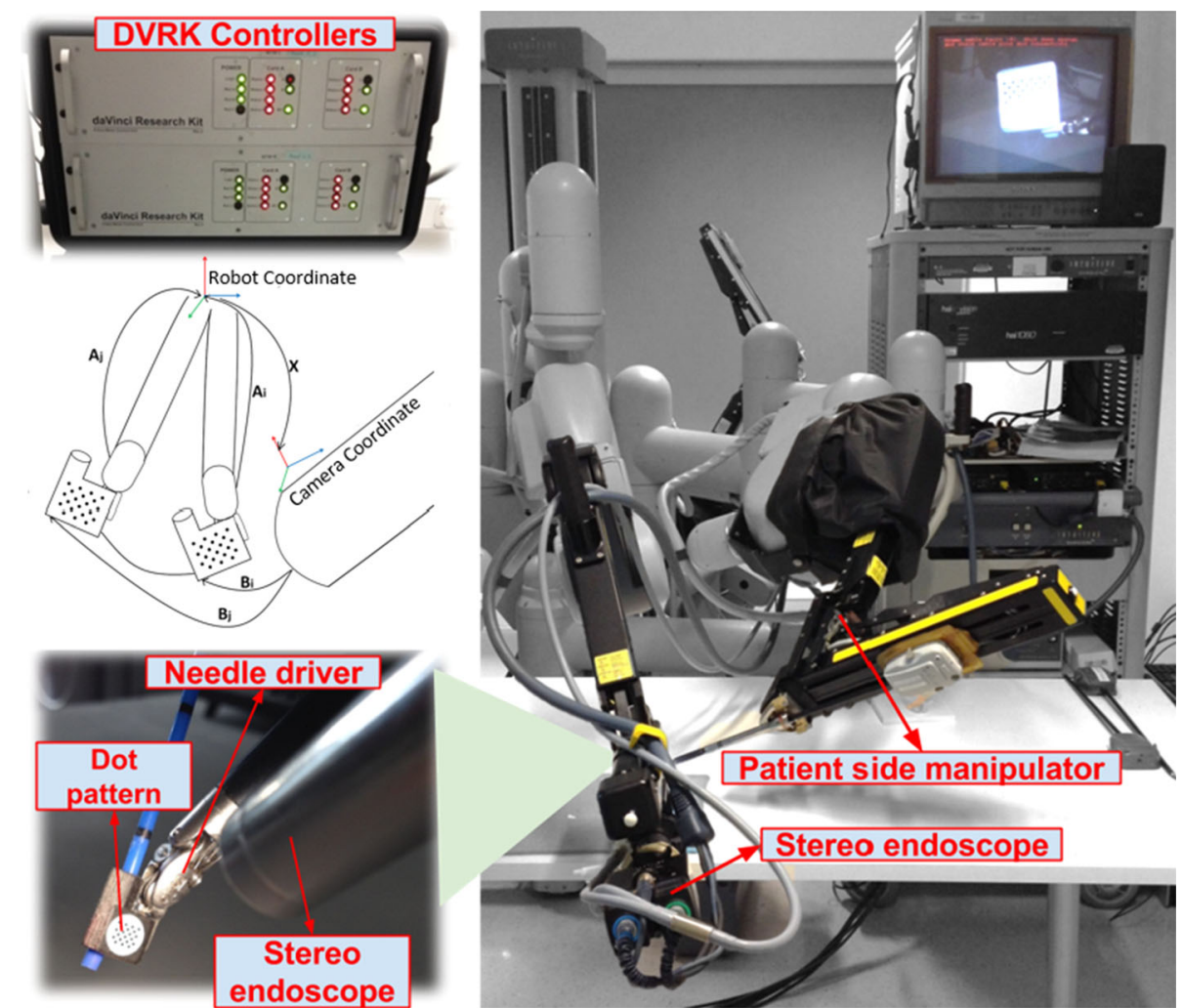

Similar to Eq. (40), we can have

$$
\begin{aligned}
& \left\|H_{l} \cdot q_{r, X}^{n}-H_{r} \cdot q_{d, X}^{n}\right\| \\
& \leqslant \|\left(H_{l} \cdot H_{l}^{+} \cdot H_{r} \cdot q_{d, X}^{n-1}\right. \\
& \left.\quad \cdot\left(H_{r} \cdot q_{d, X}^{n}\right)^{+}-I\right) H_{r} \cdot q_{d, X}^{n} \| \\
& =\left\|H_{l} \cdot H_{l}^{+} \cdot H_{r} \cdot q_{d, X}^{n-1}-H_{r} \cdot q_{d, X}^{n}\right\| \\
& =\left\|H_{l} \cdot q_{r, X}^{n-1}-H_{r} \cdot q_{d, X}^{n}\right\|
\end{aligned}
$$

\section{Experimental and simulation results}

In order to evaluate the performance of the proposed handeye calibration algorithm, detailed simulation and laboratory experiments were carried out. The simulation study was based on the Monte Carlo method, which was carried out on a workstation with $3.40 \mathrm{GHz}$ Intel Core i7 processor and $16 \mathrm{G}$ RAM. For the experimental results presented in this paper, as shown in Fig. 2, the hand-eye data were collected using the da Vinci Research Kit (DVRK) which included mechanical components from the first-generation da Vinci robotic surgical system [37] and controllers/software APIs developed by Johns Hopkins University [38]. A stereo laparoscope held by a robotic arm was used to capture various poses of a calibration pattern that contains 21 key dots. The pattern is grasped by a surgical needle driver tool that is controlled by a patient side manipulator (PSM). During the experiment, the pattern was moved to different position and orientation of the robot's workspace. The pose of the tool in the robot base coordinate was obtained via the robot forward kinematics, while a corresponding pattern pose in the camera coordinate was derived using a pair of stereo images containing the pattern.

\section{Simulation study}

In practical experiments, it is difficult to know the ground truth of the rigid transformation between camera coordinate system and robot coordinate system; therefore, we resort to simulation study with known parameters. In this simulation, the rotation part of a rigid transformation $X$ was randomly set by the $Z Y X$ convention Euler angles as roll $-0.7309 \mathrm{rad}$, pitch $0.0513 \mathrm{rad}$ and yaw $-2.0804 \mathrm{rad}$, while the translational vector was set as $[0.7822,0.1513,-0.4811]^{\mathrm{T}}$ (unit meter). Thus, the random rigid transformation $X$ can be written as:

$$
X=\left[\begin{array}{cccc}
0.7436 & -0.6667 & -0.0513 & 0.7822 \\
-0.3590 & -0.3333 & -0.8718 & 0.1513 \\
0.5641 & 0.6667 & -0.4872 & -0.4811 \\
0 & 0 & 0 & 1
\end{array}\right]
$$


and 5 pairs of $A_{i}$ and the corresponding $B_{i}$ were then generated based on the $X$ within a space of $0.25 \mathrm{~m} \times 0.25 \mathrm{~m} \times 0.25 \mathrm{~m}$.

To simulate the orientation estimation error in the $A_{i}$, a random selected $3 \times 1$ vector $\delta v_{i}$ with less than 0.035 magnitude [(to make sure the rotation angle is less than our empirical value of $0.0349 \mathrm{rad}\left(2^{\circ}\right)$ ] was applied to generate a small rotational error matrix $\delta R_{i}$ for each $R_{A_{i}}$ as:

$\delta R_{i}=\left\lfloor\delta v_{i} \times\right\rfloor+I$

Thus, the $R_{A_{i}}$ used in our simulation is

$R_{A_{i}}=\delta R_{i} R_{A_{i}}$.

Similarly, a small rotation error was also added to $R_{B_{i}}$ using the same method. To simulate the translation error as well, a zero mean noise with standard deviation $0.002 \mathrm{~m}$ was added to both $t_{A_{i}}$ and $t_{B_{i}}$.

Figure 3 shows the iterative results for the estimation of the rigid transformation $X$ : Fig. 3a gives the rotational results given by the $Z Y X$ Euler angles, while Fig. $3 \mathrm{~b}$ provides the translation vector estimation results. For systematic comparison, we also implemented the SQP algorithm to optimize the constrained problem in Eq. (5), and the results derived from the SQP algorithm are also shown in Fig. 3. As we can see from the figure, both methods can generate the $R_{X}$ and $t_{X}$ estimations accurately, but the proposed method is much faster than the traditional optimization method. After 3 iterations using our method, the estimation for $X$ is almost equal to its ground truth value. Although the optimization method can also converge to the ground truth of $X$, convergence speed is much slower and it needs more than 30 iterations to achieve the same accuracy.

To better illustrate of estimation error, Fig. 4a presents the error between the $X$ estimation and its ground truth, while Fig. 4 shows the value of the cost function $\sum_{j=1}^{J} \| A_{i} X-$ $X B_{i} \|$. From the figures, it is obvious that either the estimation error or the cost function will decrease to 0 after only 3 iterations using our method. To achieve the same accuracy, the traditional optimization method needs at least 30 iterations. Furthermore, the convergence process of our method to find $X$ is much smoother. After every single iteration, the estimation of $X$ will get closer to the ground truth and the value of the cost function will get smaller. In contrast, the estimation of $X$ using the SQP method may divert from the ground truth although the value of the cost function gets smaller after some certain iterations. It has been noted that the SQP optimization method took about $2 \mathrm{~s}$ to complete all the iterations, while our method only took less than $0.1 \mathrm{~s}$ in our simulation by using the same computing hardware. In fact, the SQP algorithm usually requires to calculate the value of cost function more than 10 times within an iteration, and it also involves sophis-

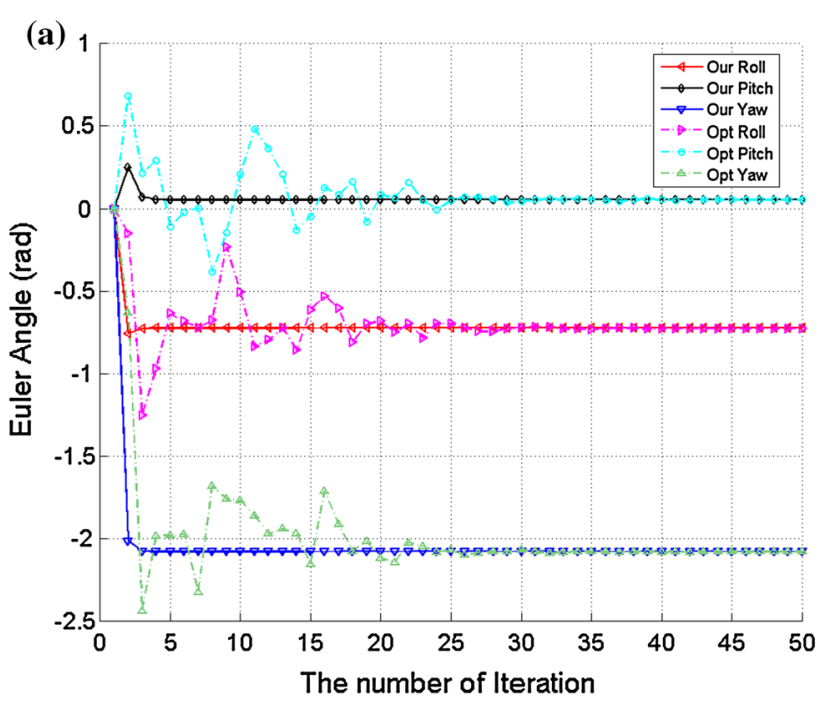

(b)

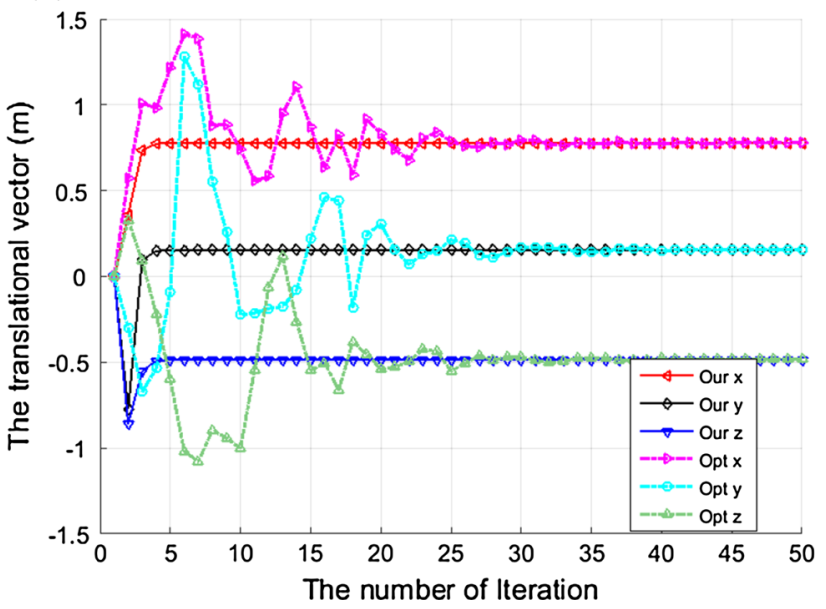

Fig. 3 The iterative results for the rigid transformation matrix $X$ estimation. a The rotational part given by the Euler angles, $\mathbf{b}$ the translational vector estimation results. It is very clear that the proposed two-step iteration method can converge much faster than the traditional optimization method

ticated Hessian and Jacobian matrix operations, which are computationally expensive. However, our proposed method only requires some basic matrix operations, such as multiplication and inverse, which therefore make our method much more efficient than the traditional optimization method. This is attractive for direct implementation of the algorithm on embedded system as part of the robotic hardware.

For this paper, the simulation was repeated for 500 times using different pairs of $A$ and $B$ (different rotation, translational, and perturbations), and statistical results for $X$ estimation are given in Table 1. It is obvious that the proposed two-step iterative method converges after 3 iterations with negligible errors, while the traditional optimization based methods needs more than iterations. In conclusion, the above analysis has shown that the proposed two-step iter- 

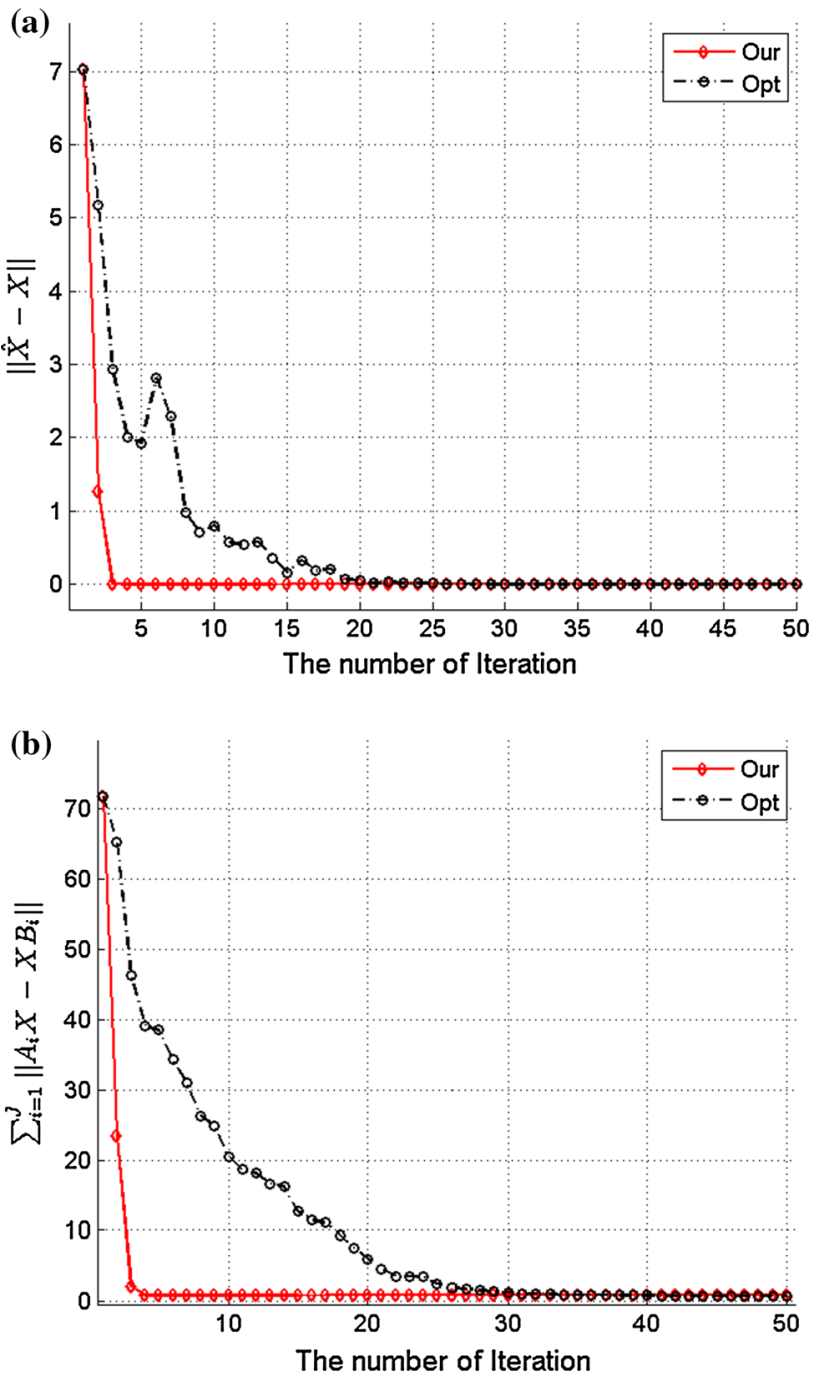

Fig. 4 The estimation error for rigid transformation $X$. a The error between the $X$ estimation and its ground truth, $\mathbf{b}$ the value of the cost function. It is very clear that the proposed two-step iteration method can converge much faster than the traditional optimization method

ation method can solve the hand-eye calibration problem accurately and efficiently.

We also noticed that when we increased rotation angle error from 0.0175 to $0.175 \mathrm{rad}$ and translation error from 0.001 to $0.01 \mathrm{~m}$ for any $A_{i}$ and corresponding $B_{i}$, both estimations of $X$ from our method and traditional optimization start to deviate from the ground truth. The main reason is the accuracy of $X$ estimation depends on the qualities of $A$ and $B$. The larger the errors in $A$ and $B$ are, the more inaccurate the $X$ estimation is.

\section{DVRK experiments}

The proposed two-step iteration method was then applied to estimating the rigid transformation between the stereo
Table 1 Iterative results over 500 simulations (shown as mean \pm STD)

\begin{tabular}{lll}
\hline & $\|X-\hat{X}\|$ & \\
\cline { 2 - 3 } & Our & Optimization \\
\hline Iteration 2 & $2.2330 \pm 9.1455$ & $5.5626 \pm 0.6195$ \\
Iteration 5 & $0.0003 \pm 0.0004$ & $1.9939 \pm 0.6316$ \\
Iteration 10 & $0.0002 \pm 0.0001$ & $1.0979 \pm 0.5014$ \\
Iteration 20 & $0.0002 \pm 0.0001$ & $0.0550 \pm 0.0358$ \\
Iteration 40 & $0.0002 \pm 0.0001$ & $0.0004 \pm 0.0002$ \\
Iteration 50 & $0.0002 \pm 0.0001$ & $0.0002 \pm 0.0001$ \\
\hline
\end{tabular}

laparoscope and the robot manipulator, as shown in Fig. 2. During the experiment, the robot manipulator was randomly moved to different positions and orientations within the robot work space, and the pose information was then extracted from the robot forward kinematics. Meanwhile, the pose information in the camera coordinate was also derived from the stereo images containing the 21 dots pattern. Eight data sets have been acquired, and in each data set, the robot manipulator was randomly placed at 5 different orientations and positions to generate 5 pairs of $A$ and $B$. Figure 5 shows the hand-eye calibration results based on the 8 independent data sets. As we can see from the figure, either the Euler angles or the translational vector estimation results are similar with very small deviation throughout all the trials performed, and the deviations are also very small. The consistency among all the 8 trials indicates the good repeatability of the proposed method. Similarly, the traditional optimization method can also get the comparable results, except it is more computationally expensive. It is also worth mentioning that although there is no ground truth for the rigid transformation between the stereo laparoscope and the robot manipulator, the consistency of the data illustrates the robustness and reproducibility of our proposed method.

After applying the proposed two-step iterative method to determine the rigid transformation between the stereo laparoscope and the robot manipulator, we then transformed the poses in the robot coordinate system into the camera system. In this experiment, we moved the robot manipulator to different positions and rotations. Similar to the last experiment, the continuous pose information in the robot frame was acquired from the robot forward kinematics, while the corresponding pose information in the camera coordinate was derived from the stereo images. We then converted the poses in the robot coordinate system into the camera system using the estimated $X$, and compared the differences. Figure 6 shows the transformation results: the red solid line indicates the poses extracted from the stereo images directly, the blue dash-dotted line indicates the poses derived from the robot forward kinematics, the cyan dashed line represents the convention using the $X$ estimated by the proposed method, while the black dotted 


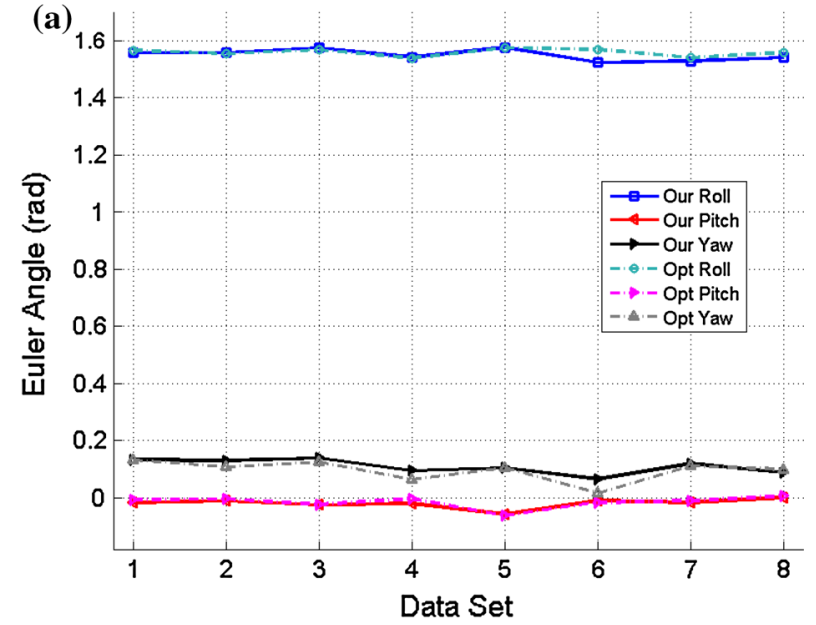

(b)

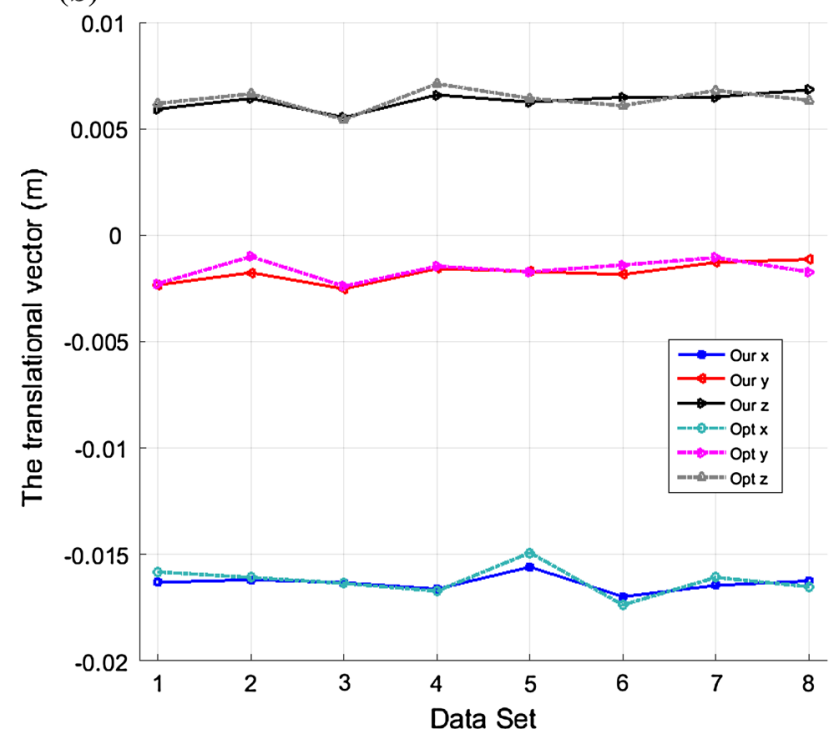

Fig. 5 The rigid transformation estimation results using the DVRK: a the rotation estimation results, $\mathbf{b}$ the translation estimation results. During the experiments, the same calibration method was repeated 8 times using different data sets. Although there is no ground truth for the rigid transformation, the estimation results have shown good consistency, which illustrates the robustness of our proposed method

line marks the transformation using the $X$ derived from the traditional optimization method.

As shown in Fig. 6, either the Euler angles or the translation vector can have significant differences for the poses given in the robot coordinate system and the camera system; therefore, hand-eye calibration must be completed to find the rigid transformation between them in advance. It is also clear that the proposed two-step iterative method can determine the rigid transformation between the stereo laparoscope and the robot manipulator and project pose information in the robot coordinate system into the camera system accurately. We also noticed that although the convergence speeds of optimization based methods are much slower than our proposed iterative
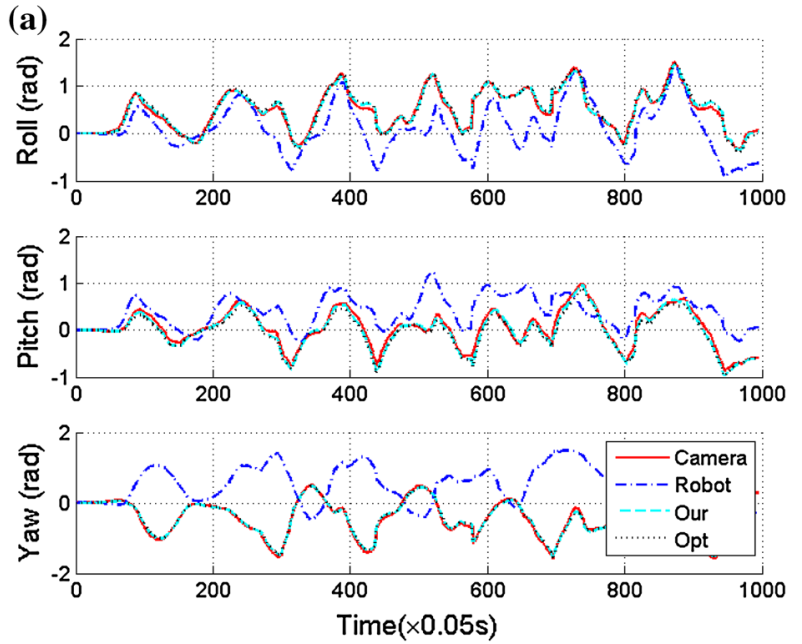

(b)
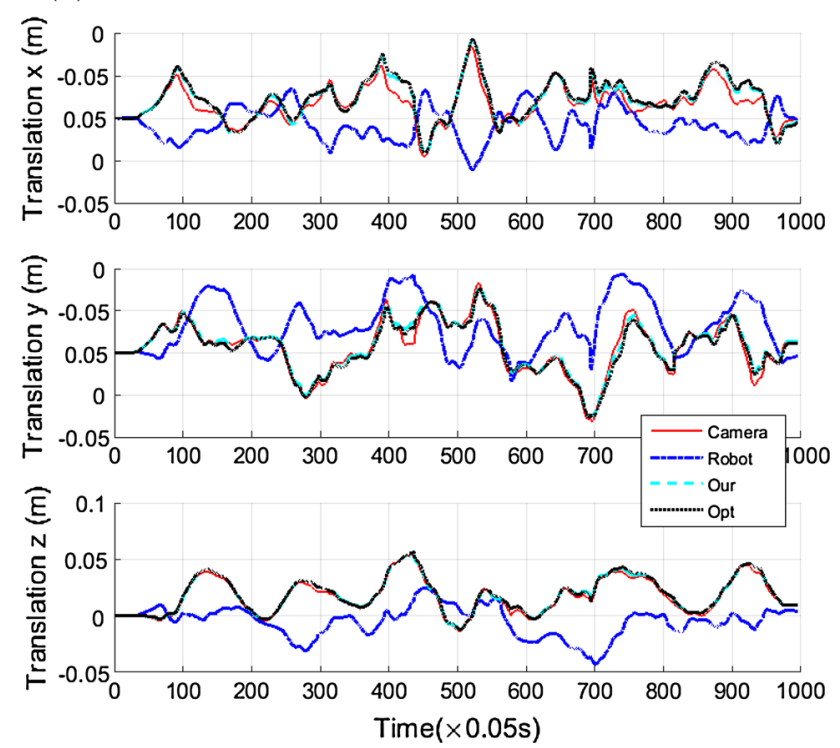

Fig. 6 The projection of poses in the camera coordinate system into the robot system. a The projection results for the rotational part, $\mathbf{b}$ the projection results of the translational part. The projection was completed using the $X$ estimated from our two-step iteration method and the traditional optimization method, respectively

method, they can also provide accurate sensor conversion. To better illustrate the advantage of the proposed two-step iteration method to solve the hand-eye calibration problem, the quantitative comparison results of the projection are also provided, as shown in Table 2. The projection using the $X$ estimated by the proposed method is slightly better than the transformation using the $X$ derived from the traditional optimization method. In general, our method has smaller RMS error and better correlation. In order to visualize the errors of the hand-eye calibration using the proposed method, Fig. 7 shows he projection of robot homogeneous transformation matrix to the image space for randomly selected three frames: 147,374 and 885 . The blue crosses indicate the projection, 
Table 2 The RMS, mean, SD and correlation coefficients of the projection of robot homogeneous transformation matrix to the image space

\begin{tabular}{|c|c|c|c|c|}
\hline & \multicolumn{2}{|c|}{ Optimization calibration } & \multicolumn{2}{|l|}{ Our calibration } \\
\hline & RMS (Mean, SD) & Correlation coefficient & RMS (Mean, SD) & Correlation coefficient \\
\hline $\begin{array}{l}\text { Roll } \\
\text { (Unit rad) }\end{array}$ & $\begin{array}{l}0.0226 \\
(-0.0198 \pm 0.0686)\end{array}$ & 0.9885 & $\begin{array}{l}0.0176 \\
(-0.0073 \pm 0.0550)\end{array}$ & 0.9914 \\
\hline $\begin{array}{l}\text { Pitch } \\
\text { (Unit rad) }\end{array}$ & $\begin{array}{l}0.0323 \\
(0.0795 \pm 0.0641)\end{array}$ & 0.9861 & $\begin{array}{l}0.0231 \\
(0.0463 \pm 0.0564)\end{array}$ & 0.9902 \\
\hline $\begin{array}{l}\text { Yaw } \\
\text { (Unit rad) }\end{array}$ & $\begin{array}{l}0.0195 \\
(-0.0196 \pm 0.0583)\end{array}$ & 0.9948 & $\begin{array}{l}0.0182 \\
(-0.0131 \pm 0.0560)\end{array}$ & 0.9952 \\
\hline $\begin{array}{l}\text { Translation } x \\
\text { (Unit mm) }\end{array}$ & $\begin{array}{l}1.2161 \\
(-2.3400 \pm 3.0459)\end{array}$ & 0.9550 & $\begin{array}{l}0.9663 \\
(-1.6659 \pm 2.5573)\end{array}$ & 0.9663 \\
\hline $\begin{array}{l}\text { Translation } y \\
\text { (Unit mm) }\end{array}$ & $\begin{array}{l}0.7859 \\
(0.3183 \pm 2.4621)\end{array}$ & 0.9804 & $\begin{array}{l}0.7773 \\
(-0.7218 \pm 2.3468)\end{array}$ & 0.9821 \\
\hline $\begin{array}{l}\text { Translation } y \\
\text { (Unit mm) }\end{array}$ & $\begin{array}{l}0.6594 \\
(-1.5204 \pm 1.4232)\end{array}$ & 0.9960 & $\begin{array}{l}0.5828 \\
(-1.1235 \pm 1.4580)\end{array}$ & 0.9956 \\
\hline
\end{tabular}
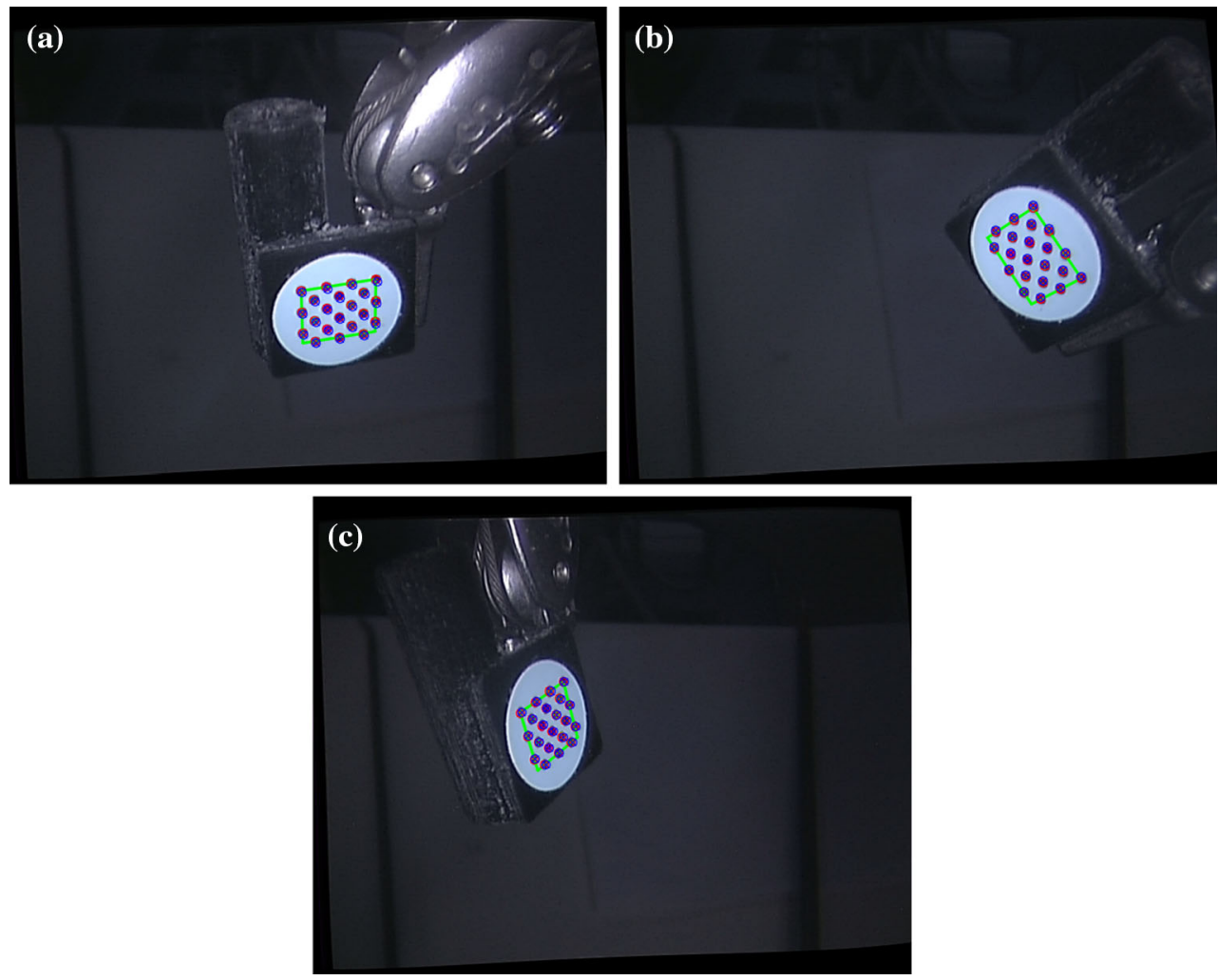

Fig. 7 The projection of robot homogeneous transformation matrix to the image space for randomly selected three frames: 147, 374 and 885. The blue crosses indicate the projection, while the red ones represent

while the red ones represent true dot locations. It can be seen that the re-projection errors are very small. In summary, the above analyses illustrate the fact that the proposed two-step iteration method can solve the hand-eye calibration problem and determine the rigid transformation between the stereo laparoscope and the robot manipulator accurately and efficiently. the dots detected in the image space directly for homogeneous transformation matrix derivation in the camera space. It shows that the errors between the projection and direct detection are very small

\section{Continuous tracking experiment}

One of the main advantages of the proposed two-step iteration method is its high efficiency and fast convergence speed, which is crucial for online hand-eye calibration. During a robot-assisted surgery, the stereo laparoscope can be moved for better field of view and visualization. This will invali- 

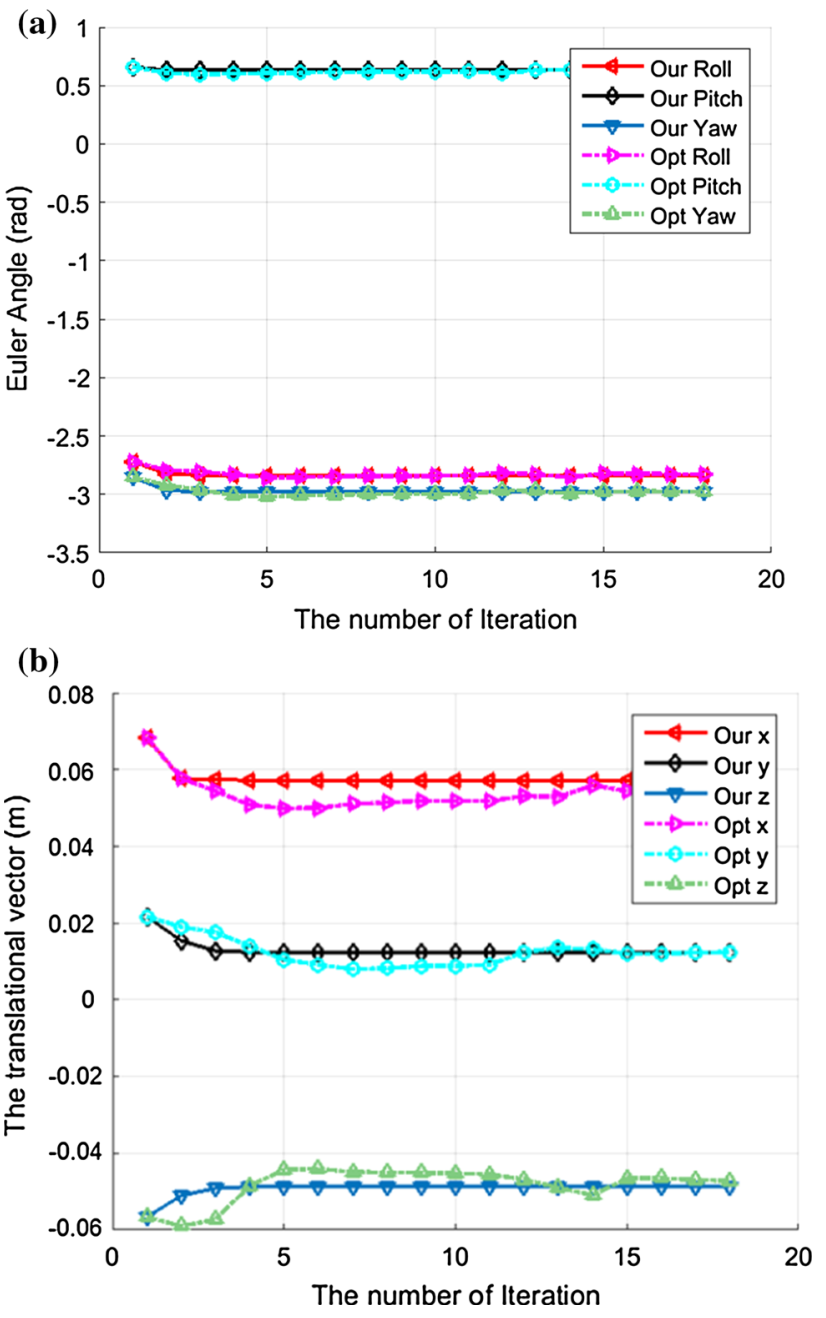

Fig. 8 The iterative results for the re-calibration after the laparoscope was shifted for a couple of millimeters. a The rotational part given by the Euler angles, $\mathbf{b}$ the translational vector estimation results. It is very clear that the proposed two-step iteration method can converge much faster than the traditional optimization method

date the hand-eye calibration, and an in-vivo re-calibration procedure is required without affecting a surgeon's workflow. To simulate this scenario, the laparoscope was moved for a couple of millimeters to introduce small change in calibration parameter $X$ after the robot and laparoscope was registered via the hand-eye calibration in our last experiment. The previous calibration result was used as the initial value to re-perform the hand-eye calibration. Figure 8 shows the re-calibration results using two sets of new poses, and the proposed two-step iterative method only needs two to three steps to converge, while the traditional optimization method requires almost 15 steps, particularly for the translational part to converge.

It should be noted that key dot calibration pattern was used to extract the new poses in the camera coordinate system, which may only be applicable to limited number of surgical

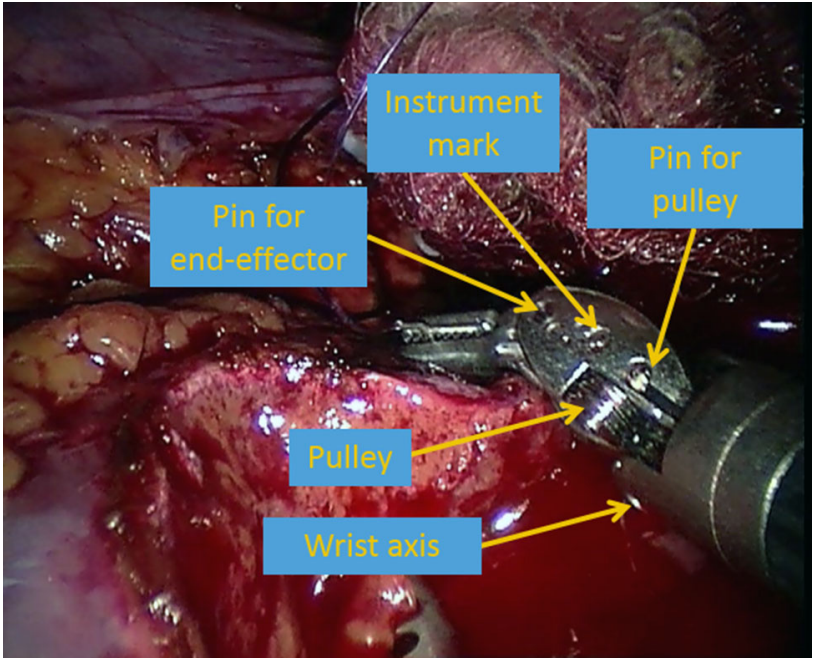

Fig. 9 The illustration of feature points on a instrument that can be applied extract the new pairs of $A$ and $B$

applications. To apply the proposed method on more general surgical tasks, markerless tracking techniques, such as instrument feature points tracking $[39,40]$ as shown in Fig. 9, are needed. Although Pachtrachai et al. [41] had successfully applied robotic surgical instruments with well-known geometry as the calibration object for hand-eye calibration, how to accurately and robustly extract the new poses based on the feature point in the presence of occlusion and stained instrument is yet to be solved. We will also evaluate the benefits of fast online calibration in robot-assisted surgery when we have positive progress in surgical instruments feature points tracking.

\section{Conclusion}

In conclusion, we have presented a computationally efficient method to solve the hand-eye calibration equation $A X=X B$ given several pairs of rigid transformations $A$ and the corresponding $B$. In our method, dual quaternion was introduced to represent the rigid transformation, and a two-step iterative method was then proposed to recover the real part and dual part of the dual quaternion simultaneously, which could thus be applied to estimate rotation and translation for the rigid transform. The proposed method was applied to determine the rigid transformation between the stereo laparoscope and the robot manipulator. Promising experimental and simulation results were achieved to illustrate the effectiveness and efficiency of the proposed method.

Our future work will investigate how to accurately and robustly extract the new poses based on the feature point in 
the presence of occlusion and stained instrument, and apply the proposed method in vivo.

\section{Compliance with ethical standards}

Conflicts of interest Zhiqiang, Lin Zhang and Guang-Zhong Yang declare that they have no conflict of interest.

Ethical approval This article does not contain any studies with human participants or animals performed by any of the authors.

Informed consent This article does not contain patient data.

Open Access This article is distributed under the terms of the Creative Commons Attribution 4.0 International License (http://creativecomm ons.org/licenses/by/4.0/), which permits unrestricted use, distribution, and reproduction in any medium, provided you give appropriate credit to the original author(s) and the source, provide a link to the Creative Commons license, and indicate if changes were made.

\section{References}

1. Bergeles C, Yang G-Z (2014) From passive tool holders to microsurgeons: safer, smaller, smarter surgical robots. IEEE Trans Biomed Eng 61(5):1565-1576

2. Du X, Allan M, Dore A, Ourselin S, Hawkes D, Kelly JD, Stoyanov D (2016) Combined 2D and 3D tracking of surgical instruments for minimally invasive and robotic-assisted surgery. Int J Comput Assist Radiol Surg 11(6):1109-1119

3. Fard MJ, Ameri S, Chinnam RB, Ellis RD (2017) Soft boundary approach for unsupervised gesture segmentation in robotic-assisted surgery. IEEE Robot Automation Lett 2(1):171-178

4. Herrell SD, Galloway RL Jr, Miga MI (2015) Image guidance in robotic-assisted renal surgery. In: Liao JC, Su L-M (eds) Advances in image-guided urologic surgery. Springer, Berlin, pp 221-241

5. Nadeau C, Krupa A (2013) Intensity-based ultrasound visual servoing: modeling and validation with $2-\mathrm{D}$ and $3-\mathrm{D}$ probes. IEEE Trans Robot 29(4):1003-1015

6. Vitiello V, Lee S-L, Cundy TP, Yang G-Z (2013) Emerging robotic platforms for minimally invasive surgery. IEEE Rev Biomed Eng 6:111-126

7. Adebar T, Fletcher A, Okamura A (2014) 3-D ultrasound-guided robotic needle steering in biological tissue. IEEE Trans Biomed Eng 61(12):2899-2910

8. Nadeau C, Ren H, Krupa A, Dupont P (2015) Intensity-based visual servoing for instrument and tissue tracking in 3D ultrasound volumes. IEEE Trans Automation Sci Eng 12(1):367-371

9. Miga MI (2016) Computational modeling for enhancing soft tissue image guided surgery: an application in neurosurgery. Ann Biomed Eng 44(1):128-138

10. Shiu YC, Ahmad S (1989) Calibration of wrist-mounted robotic sensors by solving homogeneous transform equations of the form $\mathrm{ax}=\mathrm{xb}$. IEEE Trans Robot Automation 5(1):16-29

11. Park FC, Martin BJ (1994) Robot sensor calibration: solving $a x=x b$ on the euclidean group. IEEE Trans Robot Automation 10(5):717

12. Chou JC, Kamel M (1991) Finding the position and orientation of a sensor on a robot manipulator using quaternions. Int J Robot Res 10(3):240-254

13. Liang R-h, Mao J-F (2008) Hand-eye calibration with a new linear decomposition algorithm. J Zhejiang Univ Sci A 9(10):1363-1368
14. Pan H, Wang NL, Qin YS (2014) A closed-form solution to eye-to-hand calibration towards visual grasping. Ind Robot Int $\mathrm{J}$ 41(6):567-574

15. Najafi M, Afsham N, Abolmaesumi P, Rohling R (2014) A closedform differential formulation for ultrasound spatial calibration: multi-wedge phantom. Ultrasound Med Biol 40(9):2231-2243

16. Lu Y-C, Chou JC (1995) Eight-space quaternion approach for robotic hand-eye calibration. In: Systems, man and cybernetics. IEEE international conference on intelligent systems for the 21 st century, vol 4. IEEE, pp 3316-3321

17. Daniilidis K (1999) Hand-eye calibration using dual quaternions. Int J Robot Res 18(3):286-298

18. Zhao Z, Liu Y (2009) A hand-eye calibration algorithm based on screw motions. Robotica 27(02):217-223

19. Andreff N, Horaud R, Espiau B (1999) On-line hand-eye calibration. In: 3-D Digital Imaging and Modeling, 1999. Proceedings. Second International Conference on. IEEE, pp. 430-436

20. Schmidt J, Vogt F, Niemann H (2003) Robust hand-eye calibration of an endoscopic surgery robot using dual quaternions. In: Joint pattern recognition symposium. Springer, pp 548-556

21. Strobl KH, Hirzinger G (2006) Optimal hand-eye calibration. In: IEEE/RSJ international conference on intelligent robots and systems. IEEE, pp 4647-4653

22. Mao J, Huang X, Jiang L (2010) A flexible solution to $a x=x b$ for robot hand-eye calibration. In: Proceedings of the 10th WSEAS international conference on Robotics, control and manufacturing technology. World Scientific and Engineering Academy and Society (WSEAS), pp 118-122

23. Zhao Z (2011) Hand-eye calibration using convex optimization. In: 2011 IEEE international conference on robotics and automation (ICRA). IEEE, pp 2947-2952

24. Ruland T, Pajdla T, Kruger L (2012) Globally optimal hand-eye calibration. In: 2012 IEEE conference on computer vision and pattern recognition (CVPR), pp 1035-1042

25. Ackerman MK, Cheng A, Boctor E, Chirikjian G (2014) Online ultrasound sensor calibration using gradient descent on the euclidean group. In: 2014 IEEE international conference on robotics and automation (ICRA). IEEE, pp 4900-4905

26. Heller J, Henrion D, Pajdla T (2014) Hand-eye and robot-world calibration by global polynomial optimization. In: 2014 IEEE international conference on robotics and automation (ICRA), pp 3157-3164

27. Heller J, Havlena M, Pajdla T (2016) Globally optimal handeye calibration using branch-and-bound. IEEE Trans Pattern Anal Mach Intell 38(5):1027-1033

28. Wu H, Tizzano W, Andersen TT, Andersen NA, Ravn O (2014) Hand-eye calibration and inverse kinematics of robot arm using neural network. In: Kim J-H, Matson ET, Myung H, Xu P, Karry F (eds) Robot intelligence technology and applications 2. Springer, Berlin, pp 581-591

29. Sarrazin J, Promayon E, Baumann M, Troccaz J (2015) Handeye calibration of a robot-ultrasound probe system without any 3D localizers. In: 201537 th annual international conference of the IEEE engineering in medicine and biology society (EMBC). IEEE, pp 21-24

30. Hager WW, Zhang H (2006) A new active set algorithm for box constrained optimization. SIAM J Optim 17(2):526-557

31. Nemirovski AS, Todd MJ (2008) Interior-point methods for optimization. Acta Numer 17:191-234

32. Nocedal J, Wright SJ (2006) Numerical optimization, 2nd edn. Springer Series in Operations Research and Financial Engineering, Springer, New York

33. Trawny N, Roumeliotis SI (2005) Indirect kalman filter for 3d attitude estimation. University of Minnesota, Department of Computer Science \& Engineering, Technical Report, vol 2, p 2005 
34. Zhang Z, Panousopoulou A, Yang G-Z (2014) Wearable sensor integration and bio-motion capture: a practical perspective. In: Yang G-Z (ed) Body sensor networks. Springer, Berlin, pp 495-526

35. Kenwright B (2012) A beginners guide to dual-quaternions: what they are, how they work, and how to use them for 3D character hierarchies. In: 20th international conferences on computer graphics, visualization and computer vision, pp 1-10

36. Moler CB (2004) Least squares. In: Numerical computing with Matlab, chapter 5. Society for Industrial and Applied Mathematics, pp 139-163

37. Ballantyne GH, Moll F (2003) The da vinci telerobotic surgical system: the virtual operative field and telepresence surgery. Surg Clin N Am 83(6): 1293-1304

38. Chen Z, Deguet A, Taylor R, DiMaio S, Fischer G, Kazanzides P (2013) An open-source hardware and software platform for telesurgical robotics research. In: Proceedings of the MICCAI workshop on systems and architecture for computer assisted interventions, Nagoya, pp 22-26
39. Reiter A, Allen PK, Zhao T (2014) Appearance learning for 3D tracking of robotic surgical tools. Int J Robot Res 33(2):342-356

40. Zhang L, Ye M, Chan P-L, Yang G-Z (2017) Real-time surgical tool tracking and pose estimation using a hybrid cylindrical marker. Int J Comput Assist Radiol Surg 12(6):921-930

41. Pachtrachai K, Allan M, Pawar V, Hailes S, Stoyanov D (2016) Hand-eye calibration for robotic assisted minimally invasive surgery without a calibration object. In: 2016 IEEE/RSJ international conference on intelligent robots and systems (IROS). IEEE, pp 2485-2491 